\title{
BEMs to evaluate interface cracks
}

\author{
P. Brož \\ Professional Association for Science, Research and Consultancy, \\ Czech Republic
}

\begin{abstract}
Boundary element approach to the $3 \mathrm{D}$ analysis of bimaterial interface cracks is put on. $J$-integral and stress intensity factors are calculated along the crack face employing the Energy Domain Integral and the $M_{l}$-integral techniques. The setup numerical means is employed to analyze the problem of a fibre/matrix interface crack subject to lateral loading so as to evaluate the factors of the issues obtained from two-dimensional simulations. The obtained outcomes demonstrate the important role played by the elastic properties of the fibre, the matrix and the laminate in the mixed mode fracture condition. Numerical tests performed indicate that the multi-domain procedure is suitable for bimaterial stress analysis, and as well as competent to yield precise results of the stress intensity factors $K_{\mathrm{I}}$ and $K_{\mathrm{II}}$. The multi-domain boundary contour method with possible applications to interface and dissimilar material problems and also a technique tackling with a general type of displacement and traction compatibility conditions are introduced.
\end{abstract}

Keywords: bimaterial plate, debonding, energy domain integral, interface crack, normalized SIF, shared node, virtual crack extension.

\section{Introduction}

Composite materials have highly anisotropic properties, with superb stiffness and strength behaviour in the fibre direction and rather weak properties in the transverse direction. For this reason, laminates are used being made up of several stacked plies with different fibre orientations, causing sufficient stiffness in more than one direction. Nevertheless, mechanical loading of such structure also induces loads in arbitrary directions not the same either with those of the fibre or the applied load. The reason of early failure of the transverse ply is the material heterogeneity. It has been demonstrated that a global strain of $1 \%$ already 
produces local strains of more than 5\%. An irregular packing of fibres owing to the inhomogeneous fibre distribution, increases the effect. Due to this local stress concentration, the macroscopic transverse failure strain is, in the majority of cases, extensively lower than the longitudinal failure strain. In all loading directions, the macroscopic characteristics of unidirectional fibre-reinforcedcomposite materials are hard affected by the events being present at the fibres scale. In longitudinal as well as in transverse direction, the stress transfer from the matrix to the fibres determines the mechanical behaviour of the composite, for the failure process is principally established by the strength of the fibre/matrix adherence. The stress concentrations around the fibres end in microcrack initiation that will grow along a ply to come to be a complete lateral crack.

Recently, broad analyses have been performed and many questions regarding the mechanics of interface fracture have been solved. But still, progress has been generally oriented on the two-dimensional idealization of an interface crack, and restricted endeavour has been conducted on the three-dimensional concept of interface fracture. This is partly owing to the extreme complexity of such problems and very large computational efforts required for their numerical analysis. However, given the material mismatch at the interface boundary, it goes without saying that the three-dimensional effect plays a more outstanding role in a biomaterial structure than in a homogenous one.

Due to the inherent characteristics of the BEM formulation, it provides very precision issues for problems including strong geometrical discontinuities. This makes BEM a powerful numerical means to simulate crack problems.

BEM has been far-reachingly employed to analyze an amount of problems involving two-dimensional interface cracks [1,2]. There are yet no reported results for three-dimensional problems, with the exception of an in-depth and superior study [3]. Though many authors suggest displacement and stress extrapolation methods to stipulate stress intensity factors from BEM outcomes $[4,5], J$-integral methods make up a more strong approach. As an energy concept, $J$-integral methods eliminate the need to solve local crack tip fields precisely, because if integration domains are defined over a relatively large part of the mesh, accurate modelling of the crack tip is superfluous, since the contribution to $J$-integral of the crack tip fields is not meaningful. At the same time, BEM is especially appropriate for the evaluation of path independent integrals, as the required stresses, strains and derivatives of displacements at internal points can be directly obtained from their boundary integral representations. It has been also shown that BEM yields more accurate stresses and strains at internal points when comparing with other numerical techniques, therefore better results can be achieved. Use of the $J$-integral methodology for two-dimensional interface cracks can be searched in the work by Miyazaki et al. [6].

From the available algorithms for the numerical computation of the $J$-integral in three dimensions, the Energy Domain Integral (EDI) has been frequently used to employ. Together with the EDI, the interaction or $M_{l}$-integral methodology due to Chen and Shield [7] is used in this work, in compliance with [3], for decoupling the $J$-integral into the mixed-mode stress intensity factors. The $M_{I^{-}}$ 
integral methodology is grounded on the superposition of two equilibrium states, formulated by the current problem and a set of auxiliary known solutions. The procedure has been recently announced in a number of papers using FEM to calculate stress intensity factors along three-dimensional interface cracks $[8,9]$. In the field of fracture mechanics the solution to the applications of very interest is facilitated by using a multi-domain technique grounded on the boundary contour method [15].

\section{$2 J$-integral and stress intensity factor calculation}

Take a three-dimensional crack face with a continuously turning tangent as depicted in Fig. 2(a). Characterize a local coordinate system $x^{*}$ at position $\eta$, where the crack energy release rate is evaluated, given by $x_{1}^{*}$ normal to the crack front, $x_{2}^{*}$ normal to the crack plane, and $x_{3}^{*}$ tangent to the crack front.

The energy release rate $G(\eta)$ owing to crack extension in its own plane along a three-dimensional crack front reads

$$
G(\eta)=\lim _{C \rightarrow 0} \xi_{k}(\eta) \int_{C(\eta)}\left(w \cdot \delta_{k i}-\sigma_{i j}^{*} u_{j, k}^{*}\right) n_{i} \mathrm{~d} C
$$

where $w$ is the strain energy density, $\sigma_{i j}^{*}$ and $u_{j, k}^{*}$ are Cartesian components of stress and displacement derivatives expressed in the local system $x^{*}, \xi_{k}(\eta)$ is the unit outward normal to the crack front in the local crack plane $x_{1}^{*}-x_{3}^{*}, n_{\mathrm{i}}$ is the unit vector normal to the contour $C(\eta)$ (which lies in the $x_{1}^{*}-x_{2}^{*}$ plane), and $\mathrm{d} C$ is the differential of the arc length $C$. Although eqn (1) comes from a twodimensional analysis, it applies for the three-dimensional case, in the limit as $\mathrm{C} \rightarrow 0$, plain strain conditions predominate three-dimensional fields approach to the plane problem.

So as to deduce the equivalent domain representation of eqn (1), we consider a small segment $L_{\mathrm{c}}$ of the crack front that lies in the local $x_{1}^{*}-x_{3}^{*}$ plane. Next we suppose that the segment undergoes a virtual crack advance in the plane of the crack, and we define the magnitude of the advance at each point $\eta$ as $\Delta a(\eta)$. We note that $\Delta a(\eta)$ varies continuously along $L_{\mathrm{c}}$ and disappears at each end of the segment. We have

$$
G(\eta)=\int_{L_{c}} G(\eta) \Delta a(\eta) \mathrm{d} \eta
$$

where $\mathrm{G}(\eta)$ is the integral defined in eqn (1). When $\mathrm{G}(\eta)$ is part of the point-wise energy release rate, $\bar{G}$ gives the total energy released when the finite segment $L_{\mathrm{c}}$ experinces the virtual crack advance.

The relevant domain shape of the point-wise crack-tip contour integral can be obtained from eqn (2) by considering a tubular domain $V$ surrounding the crack 
segment (see Fig. 3). As shown in the figure, the surface $S_{\mathrm{t}}$ is formed by translating the contour $C$ along the segment $L_{\mathrm{c}}$, and $S_{\mathrm{o}}$ stands for the outer surface of $V$ including the ends. Next an auxiliary function $q$ is introduced, which is sufficiently smooth in $V$ and it is determined on the surfaces of $V$ in the form:

$$
q_{k}=\left\{\begin{array}{lc}
\Delta a(\eta) \cdot \xi_{k}(\eta), & \text { on } S_{t}, \\
0, & \text { on } S_{o}
\end{array}\right.
$$

Lastly, in the limit as the tubular surface $S_{\mathrm{t}}$ is thrusted onto the crack segment $L_{\mathrm{c}}$ and in the absence of crack face tractions, we obtain the domain integral:

$$
\bar{G}=\int_{V}\left(\sigma_{i j}^{*} u_{j, k}^{*}-w \cdot \delta_{k i}\right) q_{k, i} \mathrm{~d} V
$$

During absence of body forces the integral $\bar{G}$ according to eqn (4) reduces to the domain representation of the familiar $J$-integral. If it is supposed that $G(\eta)$ is constinuous along $L_{\mathrm{c}}$, at once it follows from eqn (2) that:

$$
J(\eta)=G(\eta)=\frac{\bar{G}}{\int_{L_{c}} \Delta a(\eta) \mathrm{d} \eta}
$$

We put on the interaction or $M_{1}$-integral methodology for decoupling threedimensional mixed-mode stress intensity factors in bimaterial interface cracks. So as to make sure the purpose of the stress intensity factors we must consider the linear elastic solution of the open model of interface cracks. It is assumed in this model that the small contact zone that always develops at the crack tip is physically non-relevant. We label the material occupying the upper half-plane as material 1 with Young modulus $E_{1}$ and Poisson ratio $v_{1}$ (see Fig. 1). The material occupying the lower half-plane has Young modulus $E_{2}$ and Poisson ratio $v_{2}$. Let us consider now two equilibrium states with field variables denoted by the superscripts (1) and (2). Superposition of the two equilibrium states results in another one, $(1+2)$. Next, the stress intensity factors $K_{j}^{(1+2)}$ are equal to

$$
K_{j}^{(1+2)}=K_{j}^{(1)}+K_{j}^{(2)} \quad(j=\mathrm{I}, \mathrm{II}, \mathrm{III})
$$

The relationship between the $J$-integral and the stress intensity factors of an interface crack may be written in the form

$$
J=\frac{1}{E^{*} \cosh ^{2}(\pi \varepsilon)}\left[K_{I}^{2}+K_{I I}^{2}\right]+\frac{1}{2 \mu^{*}} K_{I I I}^{2}
$$

where $E^{*}$ and $\mu^{*}$ are the effective Young and shear modulus 


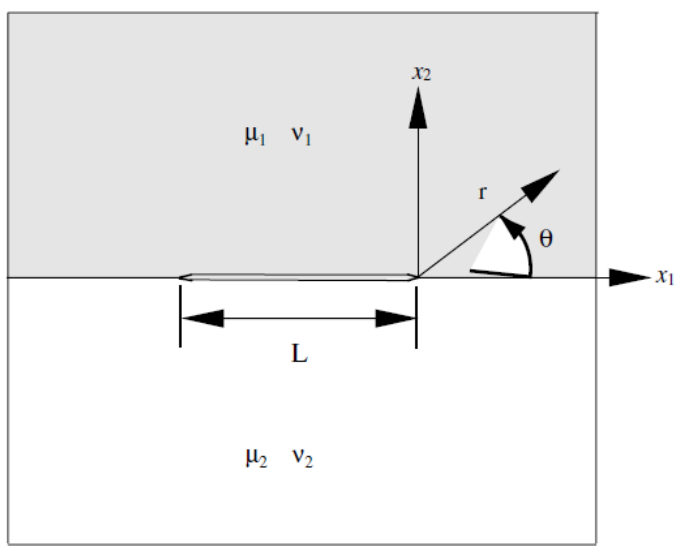

Figure 1: $\quad$ Bimaterial plate weakened by an interface crack (taken from [3]).

$$
\frac{1}{E^{*}}=\frac{1}{2}\left(\frac{1-v_{1}^{2}}{E_{1}}+\frac{1-v_{2}^{2}}{E_{2}}\right), \frac{1}{\mu^{*}}=\frac{1}{2}\left(\frac{1}{\mu_{1}}+\frac{1}{\mu_{2}}\right)
$$

and $\varepsilon$ represents the bimaterial constant

$$
\varepsilon=\frac{1}{2 \pi}\left(\frac{\kappa_{1} \mu_{2}+\mu_{1}}{\kappa_{2} \mu_{1}+\mu_{21}}\right)
$$

According to eqns (6), (7), the stress intensity factors can be related to the $J$ integral for the superimposed state $(1+2)$ leading to

$$
J^{(1+2)}=\frac{1}{E^{*} \cosh ^{2}(\pi \varepsilon)}\left[\left(K_{I}^{(1+2)}\right)^{2}+\left(K_{I I}^{(1+2)}\right)^{2}\right]+\frac{1}{2 \mu^{*}}\left(K_{I I I}^{(1+2)}\right)^{2}
$$

We may rewrite eqn (10) by virtue of the stress intensity factors for the equilibrium states (1) and (2), to pass:

$$
J^{(1+2)}=J^{(1)}+J^{(2)}+\frac{2}{E^{*} \cosh ^{2}(\pi \varepsilon)}\left[K_{I}^{(1)} K_{I}^{(2)}+K_{I I}^{(1)} K_{I}^{(2)}\right]+\frac{1}{2 \mu^{*}} K_{I I I}^{(1)} K_{I I I}^{(2)}
$$

Then, the $M_{l}$-integral is determined being

$$
M_{1}=J^{(1+2)}-J^{(1)}-J^{(2)}=\frac{2}{E^{*} \cosh ^{2}(\pi \varepsilon)}\left[K_{I}^{(1)} K_{I}^{(2)}+K_{I I}^{(1)} K_{I}^{(2)}\right]+\frac{1}{2 \mu^{*}} K_{I I I}^{(1)} K_{I I I}^{(2)}
$$

In compliance with eqn (4), a domain representation of the $M_{1}$-integral can be obtained in the following form: 


$$
M_{1}=\int_{V}\left(\sigma_{i j}^{*(1)} u_{j, k}^{*(2)}+\sigma_{i j}^{*(2)} u_{j, k}^{*(1)}-\sigma_{i j}^{*(1)} \varepsilon_{i j}^{*(2)} \delta_{k i}\right) q_{k, i} \mathrm{~d} V
$$

\section{Boundary element realization}

Relations (4) and (13) facilitate the computation of $J$-integral and the mixedmode stress intensity factors at any position $\eta$ on the crack front to be performed. In each instance, this requires the evaluation of a volume integral within closed domains that enclose a segment of the crack front $L_{\mathrm{c}}$. A natural choice here is to make $\eta$ coincident with the element nodes on the crack front, while $L_{\mathrm{c}}$ is taken as the element or element sides at which points $\eta$ lie (see Fig. 2). The portion of the model domain in which the volume integrals are evaluated is discretized using 27-noded isoparametric (brick) cells, over which stresses, strains and displacements derivatives are approximated by products of the cell interpolation functions $\Psi_{\mathrm{i}}$ and the nodal values of $\sigma_{\mathrm{ij}}, \varepsilon_{\mathrm{ij}}$ and $u_{\mathrm{i}, \mathrm{j}}$. Nodal values of this variable depend on whether the node is internal or lies on the model boundary. Volume discretization is designed to have web-style geometry around the crack front, while the integration volumes are taken to coincide with the different rings of cells, what is demonstrated in Fig. 3, where the front of the model was partially removed to point out the crack and the integration domains.

Conforming to Fig. 2, three different cases are in want of the reflection, depending on whether the node of interest $\mathrm{M}$ is in the middle of an element side (mid-side node), it is shared by two elements (shared node), or it is situated the same with the external surface (surface node). If the node $M$ is a mid-side node or surface node, $L_{\mathrm{c}}$ (the segment of the crack front over which the $M_{1}$-integral is computed) spans over one element, connecting nodes $M-1, M$, and $M+1$ and nodes $M-2, M-1$ and $M$, respectively. On the other hand, if $M$ is a shared node, $L_{\mathrm{c}}$ spans over two elements, connecting nodes from $M-2$ to $M+2$.

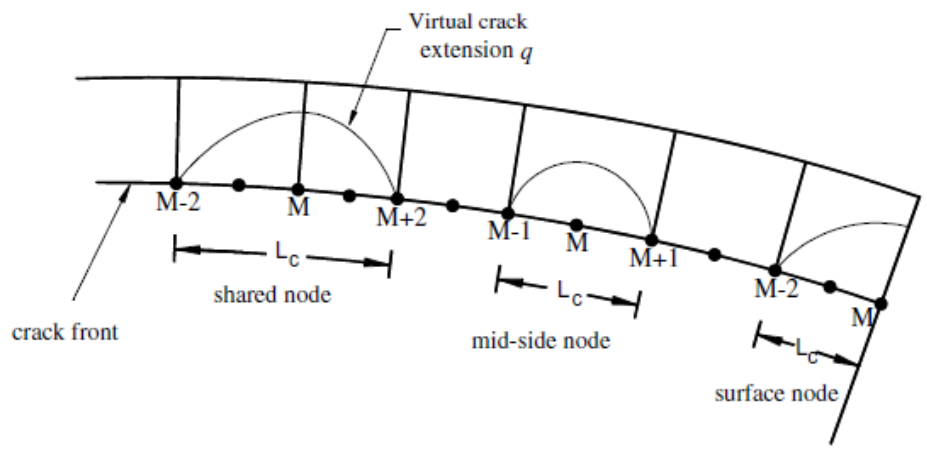

Figure 2: Scheme of the volume cells in the crack front region explaining the virtual crack stretches for a shared node, a mid-side node and a surface node [3]. 


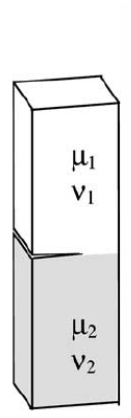

(a)

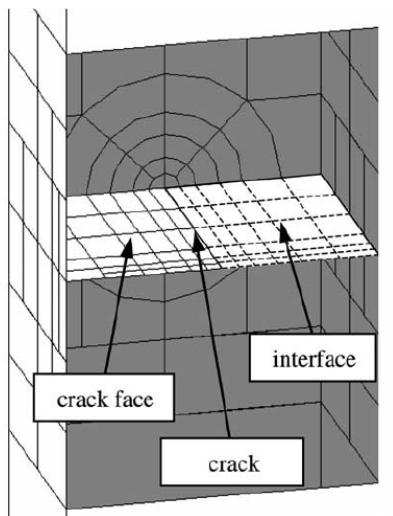

(b)

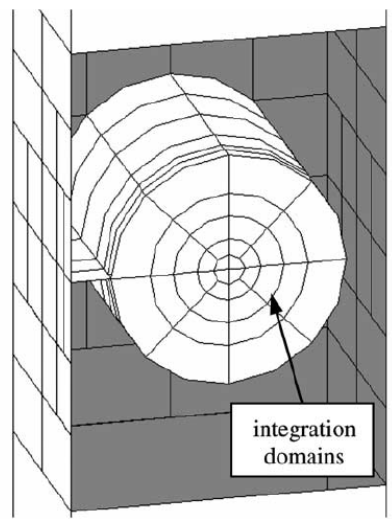

(c)

Figure 3: (a) Problem geometry, (b) boundary element discretization (c) integration domains.

\section{Explanation of a fibre/matrix interface crack subject to lateral loading}

A fibre/matrix interface crack is a special example of interface crack. Highresolution numerical BEM models [11] and analytical solutions [12] have shown that for arc-shaped debonds an extensive region with negative opening

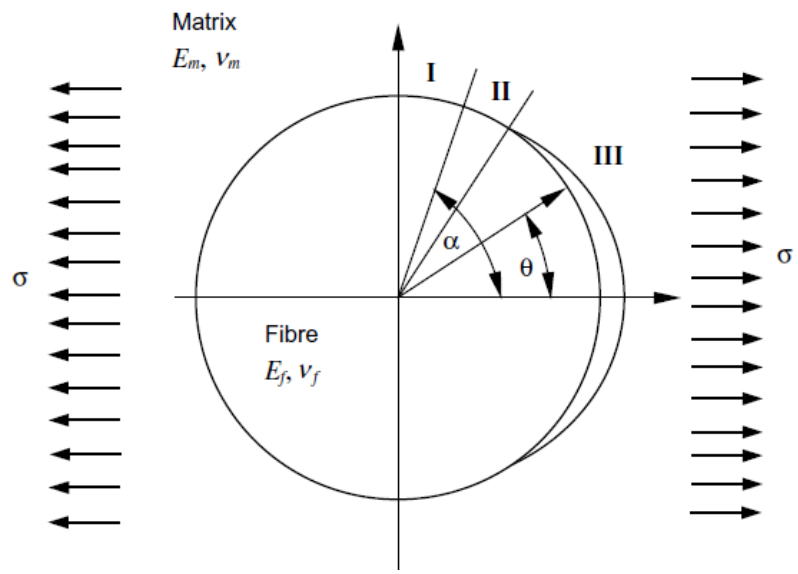

Figure 4: Schematic representation of the cross-section of the fibre embedded in matrix and loaded transverse to the fibre axis. The fibre/matrix interface is partially debonded and three regions at the interface can be distinguished: I, perfect bonding; II, debonded zone with debond face contact; III, open zone [11]. 
(overlapping) before oscillation of the solution may, depending on the debonding length, arise. The problem must be under these circumstances modelled in accordance with Comninou conclusions [13] as appears in Fig. 4 [11]. Region I represents perfect bonding between fibre and matrix whereas Regions II and III represents the debonded part, Region II corresponding to the contact zone and Region III corresponding to the opened part of the debonded zone. A physical explanation of the relative sizes of overlapping predicted by the analytical solutions and the BEM models is given in [10]. When the debonding starts to reach approximately $60^{\circ}$, a contact zone of physical meaning can be established.

\section{Discussion about results}

If the effect of the fibres in the laminate is taken into consideration (results labelled as "fibre in laminate"), the large stiffness of the specimen in the direction of the thickness causes that the crack behaves as in the plain strain model, and a constant $J$-integral value is obtained along the entire crack front. The two other sets of results are in accordance with the limiting cases for which the elastic properties of the isotropy plane are put the same to those of the matrix and the fibre. If the elastic properties are those of the matrix, the $J$-integral value is practically the same to that obtained for the single fibre example at the interior of the specimen, but it decreases at the free surface. Lastly, when the elastic properties of the isotropy plane are those of the fibre (the most rigid of all cases analyzed) $J$-integral presents its lowest level, and analogously to the homogenous case it introduces a constant value along the complete crack face.

The results facilitate the interpretation of experimental observations as those reported by Meurs [14] who tested a single glass-fibre-reinforced sample subject to lateral loading with four increasing steps when verified initiation of debonding.

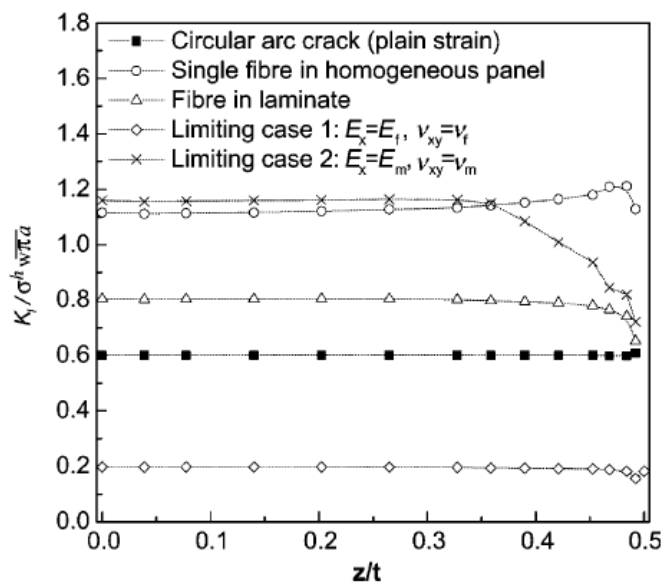

Figure 5: $\quad K_{\mathrm{I}}$ along the crack front for the fibre/matrix interface crack. 
Note that in accordance with the numerical results for the single fibre example, debond crack initiates at the specimen surface where the maximum $J$ integral value is achieved.

Stress intensity factor issues are shown for the three modes of cracking eg in Fig. 5. Results are normalized with respect to $\sigma^{\infty} \sqrt{\pi a}$. Fig. 5 permits watching that the characteristics of $K_{\mathrm{I}}$ values are obtained for the cases with the largest material mismatch between the fibre and the vicinity material, only with the exception of the limiting case for which the elastic properties of the isotropy plane are put on identical with those of the matrix, $K_{\mathrm{I}}$ results present a constant value along the majority of the crack front.

\section{Solution to biomaterial interface cracks [15]}

Problems of fracture mechanics as far as cracks at the interface between dissimilar materials are of interesting owing to their relevance to issues of debonding or delamination in composite materials. Concurrently, analytical studies (eg [16, 17, 19]) have demonstrated that the stress field close to an interface crack tip shows an oscillatory singularity. Even though the oscillation region is restricted to the vicinity of the crack tip, it has become visible to put on a challenge to the FEM and BEM, in precisely predicting the individual SIF for interface crack problems. The primary advantage of the BEM over the FEM in solving fracture problems is that only the boundary of the domain is discretized. Substantial amount of multi-domain BEM studies has been devoted to problems involving bimaterial interface cracks. The multi-domain boundary contour method or MBCM technique cas also be applied to solve bimaterial interface fracture problems where the SIFs are among interestingly parameters.

In the case of bimaterial interface cracks, the complex SIFs, gained by dint of the displacement correlation technique, are related by (see [18])

$$
K_{1}+i K_{2}=\lim _{r \rightarrow 0} \frac{2 \cosh (\pi \varepsilon)}{C} \sqrt{\frac{2 \pi}{r}}(1+2 i \varepsilon)\left(\Delta u_{n}+i \Delta u_{t}\right)\left(\frac{r}{l}\right)^{-i \varepsilon}
$$

where $\Delta u_{\mathrm{t}}$ and $\Delta u_{\mathrm{n}}$ are the tangential and normal components of the crack opening displacement, $l$ is a random length used to normalize the distance $r$ from the crack tip, and

$$
\begin{gathered}
C=\frac{\kappa_{1}+1}{G_{1}}+\frac{\kappa_{2}+1}{G_{2}} \\
\varepsilon=\frac{1}{2 \pi} \ln \left|\left(\frac{\kappa_{1}}{G_{1}}+\frac{1}{G_{2}}\right) /\left(\frac{\kappa_{2}}{G_{2}}+\frac{1}{G_{1}}\right)\right| \\
\kappa_{j}= \begin{cases}3-4 v_{j} & \text { (plane strain) } \\
\left(3-v_{j}\right) /\left(1+v_{j}\right) & \text { (plane stress) }\end{cases}
\end{gathered}
$$

with $G_{1}$ and $v_{\mathrm{j}}$ as the shear modulus and Poisson's ratio of the material $j$. 
In the context of the MBCM, the above limit is at a rough estimate evaluated by using a small value of $r$ which is the distance from the current crack tip to a nearly node on the crack-tip element. Therefore, the COD components in eqn (14) are those at the selected node, determined in the form:

$$
\begin{aligned}
& \Delta u_{t}=\Delta u_{1} \cos \theta+\Delta u_{2} \sin \theta, \\
& \Delta u_{n}=-\Delta u_{1} \sin \theta+\Delta u_{2} \cos \theta
\end{aligned}
$$

where $\theta$ is the crack bearings at the said crack tip, $\Delta u_{i}=u_{i}^{\text {upper }}-u_{i}^{\text {lower }}$ where $i=$ 1,2 , and $u_{i}^{\text {upper }}$ and $u_{i}^{\text {lower }}$ are the nodal displacement components of the upper and lower faces of the crack being specified from the MBCM analysis.

In general, it has been indicated that more accurate SIFS are obtained if the end-node of the crack-tip element is chosen for determining $r$ instead of the midnode (see Fig. 6). This may be clarified by the fact that if $r$ is too small, the numerical results are degenerated by the oscillatory stress field region near the crack tip.

For cracks in a homogenous material, $\varepsilon=0$ and $C=2(\kappa+1) / \mu$ (according to eqn (15)). As a result, eqn (14) comes to be

$$
K_{\mathrm{I}}+i K_{\mathrm{II}}=\frac{G}{\kappa+1} \sqrt{\frac{2 \pi}{r}}\left(\Delta u_{n}+i \Delta u_{t}\right)
$$

where the notations $K_{\mathrm{I}}$ and $K_{\mathrm{II}}$ are used to name the mode-I and mode-II. SIFs for homogeneous materials.

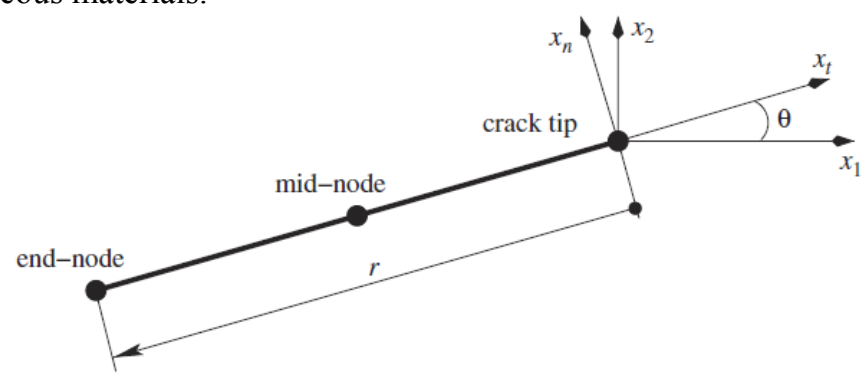

Figure 6: Crack-tip element.

\section{Conclusion}

Boundary element methodology for the three-dimensional analysis of bimaterial interface cracks has been presented. The interface crack study is spoken to, using a multidomain BEM formulation so as to be the reason for the different material properties at both sides of the crack. The parameters of fracture mechanics, namely $J$-integral and stress intensity factors, are calculated along the crack front employing the Energy Domain Integral and the $M_{1}$-integral methodologies. 
These are implemented as a post-processing technique, and so it can be applied to the results from a particular model at a later stage. The carrying out exploits the efficiency of the boundary integral equation to directly obtain the demanded displacement derivatives, stress and strain fields from their boundary integral symbolizations.

The suggested numerical aid is employed to analyze the problem of a fibre/matrix interface crack under transverse loading in order to assess its threedimensional nature. This three-dimensional model will in any case permit in the future the possibility of the interaction between longitudinal and circumferential growth of the crack to be studied. Obtained results show the leading role played by the relative elastic properties of the fibre, the matrix and the laminate on the state of mixed mode fracture. The instance of a single debonded fibre in a homogenous panel sets up the most severe condition, because it presents a large material mismatch with low lateral constraint. On the other hand, a fibre in a laminate behaves very similarly to a plain-strain case analysis, indicating that three-dimensional effects are very poor for this case. Lastly, the computed mixed-mode stress intensity factor indicates, that for most of the material combinations studied, $K_{\mathrm{I}}$ does not present important changes along the crack face, inclusive of the zone in the vicinity of the free lateral surface. By contrast, the boundary layer effect is noticeable for $K_{\mathrm{II}}$ and $K_{\mathrm{III}}$.

\section{Acknowledgement}

The author gratefully acknowledges the financial support of the presented research by the Grant Agency of the Czech Republic (project No. 103/09/1580).

\section{References}

[1] Cho, S.B., Lee, K.R., Coi, Y.S., Yuuki, R., Determination of stress intensity factors and boundary element analysis for interface cracks in dissimilar anisotropic materials. Eng Fract Mech, 43, pp. 603-614, 1992.

[2] Yuuki, R., Xu, J-Q., Boundary element analysis of dissimilar materials and interface crack. Comput Mech, 14, pp. 116-127, 1994.

[3] Cisilino, A.P., Ortiz, J.E. Three-dimensional boundary element assessment of a fibre/matrix interface crack under transverse loading. Computers and Structures, 83, pp. 856-869, 2005.

[4] Tan, C.L., Gao, Y.L., Treatment of bimaterial interface crack problems using the boundary element method. Eng Fract Mech, 36, pp. 919-932, 1990.

[5] Yuuki, R., Cho, S.B., Efficient boundary element analysis of stress intensity factors for interface cracks in dissimilar materials. Eng Fract Mech, 34, pp. 179-188, 1989.

[6] Miyazaki, N., Ikeda, T., Soda, T., Munakata, T., Stress intensity factor analysis of interface crack using boundary element method-Application of contour-integral method. Eng Fract Mech, 45, pp. 599-610, 1993. 
[7] Chen FHK, Shield RT. Conservation laws in elasticity of the J-integral type. J Appl Math Phys (ZAMP), 28, pp.1-22, 1977.

[8] Gosz, M., Dolbow, J., Moran, B., Domain integral formulation for stress intensity factor computation along curved three-dimensional interface cracks. Int J Solids Struct, 35, pp. 1763-1783, 1998.

[9] Nagashima, T., Omoto, Y., Tani, S., Stress intensity factor analysis of interface cracks using X-FEM. Int J Num Meth Eng, 56, pp. 1151-1173, 2003.

[10] Cisilino, A.P., Aliabadi, M.H., BEM implementation of the energy domain integral for the elastoplastic analysis of 3D fracture problems. Int J Fract, 96, pp. 229-245, 1999.

[11] Varna, J., París, F., del Caňo, J.C., The effect of crack-face contact on fibre/matrix debonding in transverse tensile loading. Compos Sci Technol, 57, pp. 523-532, 1997.

[12] Toya, M.A., Crack along the interface of a circular inclusion embedded in an infinite solid. J Mech Phys Solids, 22, pp. 325-348, 1975.

[13] Comninou, M., The interface crack. ASME J Appl Mech, 44, pp. 631-636, 1977.

[14] Meurs, P., Characterization of Microphenomena in transversely loaded composite material. PhD Thesis. Technische Universiteit Eindhoven, 1998.

[15] Phan, A.-V., Mukherjee, S., The multi-domain boundary contour method for interface and dissimilar material problems. Engn Anal with BE, 33, pp. 668-677, 2009.

[16] Erdogan F. Stress distribution in a nonhomogeneous elastic plane with cracks. J Appl Mech, 30, pp. 232-236, 1963.

[17] Rice, J.R., Sih, G.C., Plane problems of cracks in dissimilar media. J Appl Mech, 32, pp.418-23, 1965.

[18] Nagashima, T., Omoto, Y., Tani, S., Stress intensity factor analysis of interface cracks using X-FEM. Int J Numer Methods Eng, 56, pp. 11511173, 2003.

[19] Rice, J.R., Elastic fracture mechanics concepts for interfacial cracks. J Appl Mech, 55, pp. 98-103, 1988. 